\title{
A history of robots: from science fiction to surgical robotics
}

\author{
N. G. Hockstein · C. G. Gourin · R. A. Faust • \\ D. J. Terris
}

Received: 27 October 2006 / Accepted: 15 January 2007 / Published online: 17 March 2007

(C) Springer London 2007

\begin{abstract}
Surgical robotics is an evolving field with great advances having been made over the last decade. The origin of robotics was in the science-fiction literature and from there industrial applications, and more recently commercially available, surgical robotic devices have been realized. In this review, we examine the field of robotics from its roots in literature to its development for clinical surgical use. Surgical mills and telerobotic devices are discussed, as are potential future developments.
\end{abstract}

Keywords Robot $\cdot$ Robotics $\cdot$ Robotic surgery Minimally invasive surgery $\cdot$ daVinci $\cdot$ Zeus

N. G. Hockstein

Otolaryngology, Head and Neck Surgery,

Christiana Care Health System, Newark, DE, USA

N. G. Hockstein $(\square)$

Family Ear, Nose, and Throat Physicians,

1941 Limestone Road, Suite 210,

Wilmington, DE 19808, USA

e-mail: neilhockstein@ verizon.net

C. G. Gourin · D. J. Terris

Otolaryngology, Head and Neck Surgery,

Medical College of Georgia, Augusta, GA, USA

e-mail: cgourin@mcg.edu

D. J. Terris

e-mail: dterris@mcg.edu

R. A. Faust

Otolaryngology, Columbus Children's Hospital,

Columbus, OH, USA

e-mail: faustr@chi.osu.edu

\section{Introduction}

Robots have become incorporated into daily life over the last half century: what was once only science fiction has now become a reality. Today, everyone living in the developed world benefits from the advances in robotics in everyday life. Whereas robots have not been incorporated into daily life in the manner portrayed by the animated series "The Jetsons" (Warner Brothers Entertainment, Inc., Burbank, CA, USA), the manufacturing, service, and healthcare industries have all incorporated robotics to help improve efficiency and precision. Robots help to build our machines, package our foods, and wash our cars. While robots are commonly employed in the healthcare laboratory setting, they have been more slowly integrated into clinical medicine. Over the last two decades, research in surgical robotics has been continually increasing with a geometric rise in the number of manuscripts published each year (Fig. 1). Surgical robotics is an evolving field aiming to take advantage of the features of robotics that have made them so valuable in other industries.

\section{History}

At the dawn of the 20th century, robots were not yet a part of popular science fiction. It was not until 1917 when Joseph Capek wrote the short story Opilec describing automats and 1921 when his brother Karel Capek wrote the play Rossum's Univeral Robots (RUR) that the concept of robotics entered the popular consciousness [1,2]. Which brother originally coined the term robot is a matter of debate in the Czech literary world. The term robot is derived from the Czech word, robota, meaning serf or laborer. Karl Capek 


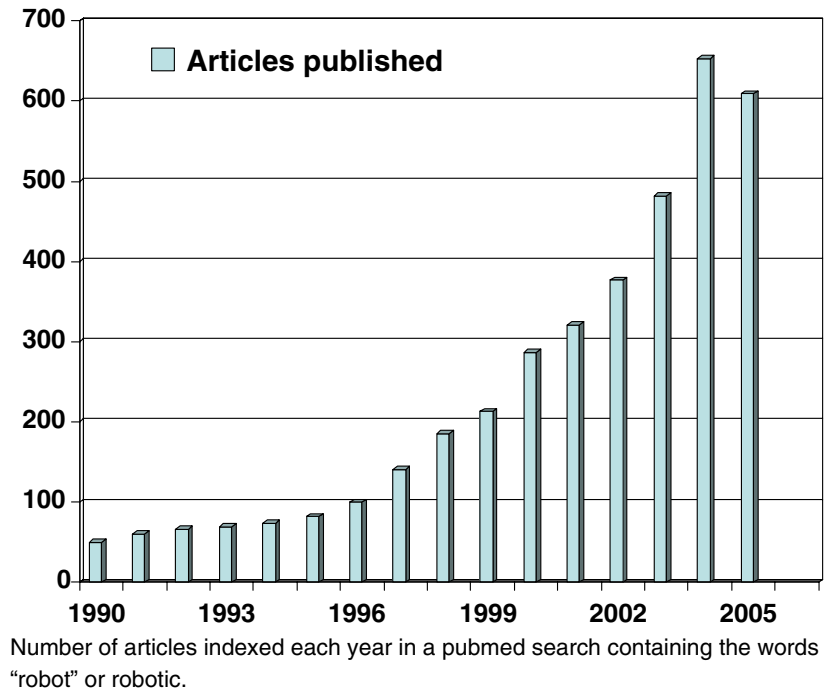

Fig. 1 Graph depicting the number of manuscripts indexed per year in a PubMed search containing the words "robot" or "robotic"

intended for his play, RUR, to protest the rapid growth of modern technology and thus he described an evolution of the robots with increasing capabilities and the eventual revolt of these robots against their human counterparts [3]. Inadvertantly, the Capek brothers introduced the term robot into modern language and sparked public fascination with their creations.

Isaac Asimov is credited with the popularization of robotics in a collection of short stories published between 1938 and 1942. Asimov is best known for his three laws governing robot behavior.

1. A robot may not injure a human being or through inaction allow a human to come to harm.

2. A robot must obey orders given it by humans except when doing so conflicts with the first law.

3. A robot must protect its own existence as long as this does not conflict with the first or second law.

Asimov conceived his laws of robotics to impose order on the free will of his fictional robots [4]. Since then, robots have been widely depicted in literary and cinematic fiction, sometimes as man's friend (Star Wars), but often as man's enemy (The Terminator). Over the last half century, robots similar to those first envisioned by Capek have become a reality and are used to perform mechanical labor in factories, in an effort to minimize human error and injury while increasing production efficiency [5].

The transition from science fiction to reality occurred in 1958 when General Motors introduced the Unimate to assist in automobile production. Since Unimate's first use on the assembly line in 1961, the application of robotics to industry has exploded [6]. Robots have since been used in a variety of applications including deep sea and space explo- ration, military use, and for search and rescue missions. In all cases, robotics has the aim of duplicating or improving upon human function or serving in roles too hazardous for direct human work.

A variety of classifications for different types of robots help to describe these heterogeneous devices. Robots can be characterized as automated arms, mobile devices, mills, or telerobotic devices. Additionally, they can be active, semiactive, or passive. Active devices are totally programmable and carry out tasks independently. One can imagine a physician entering three-dimensional (3D) computed tomography data into a computer and then programming the computer to direct a mill to remove particular areas of bone. Semiactive devices and passive robotic devices translate movements from an operator's or surgeon's hands into powered or unpowered movements of the robot end-effector arms. Surgical robots in use or research today include both active mills and semiactive telerobotic devices.

\section{Active surgical robotics}

Active robotic devices, in which pre-programmed data and computer-generated algorithms function without real-time operator input, were the first robots to be used in live surgical applications. In 1985, the first surgical application of industrial robotic technology was described when an industrial robotic arm was modified to perform a stereotactic brain biopsy with $0.05 \mathrm{~mm}$ accuracy. This served as the prototype for Neuromate (Integrated Surgical Systems, Sacramento, CA, USA) which received Food and Drug Administration (FDA) approval in 1999 [7]. In 1992, the Robodoc (Integrated Surgical Systems) was introduced for use in hip replacement surgery (Fig. 2). The Robodoc is a computer-guided mill used to core the femoral head to receive a hip replacement prosthesis. Clinical trials demonstrate greater accuracy comparing the well drilled by the Robodoc to conventional techniques. Whereas the Robodoc has been used in thousands of patients in Europe, it has not yet received FDA approval in the United States because of concerns regarding complication rates [8-10]. Similar devices have been designed for use in knee replacement and temporal bone surgery, notably the Acrobot (The Acrobot Company, Ltd., London, UK) and the RX-130 robot (Staubli Unimation Inc., Faverges, France), respectively. Neither device has yet completed clinical testing nor received FDA approval [11, 12].

\section{Telerobotics}

The concept of remote robotic operation has long been recognized to have benefits in several different fields. Defusing 
Fig. 2 The daVinci S surgical system. The surgeon's console is shown on the left and the robot arm tower on the right
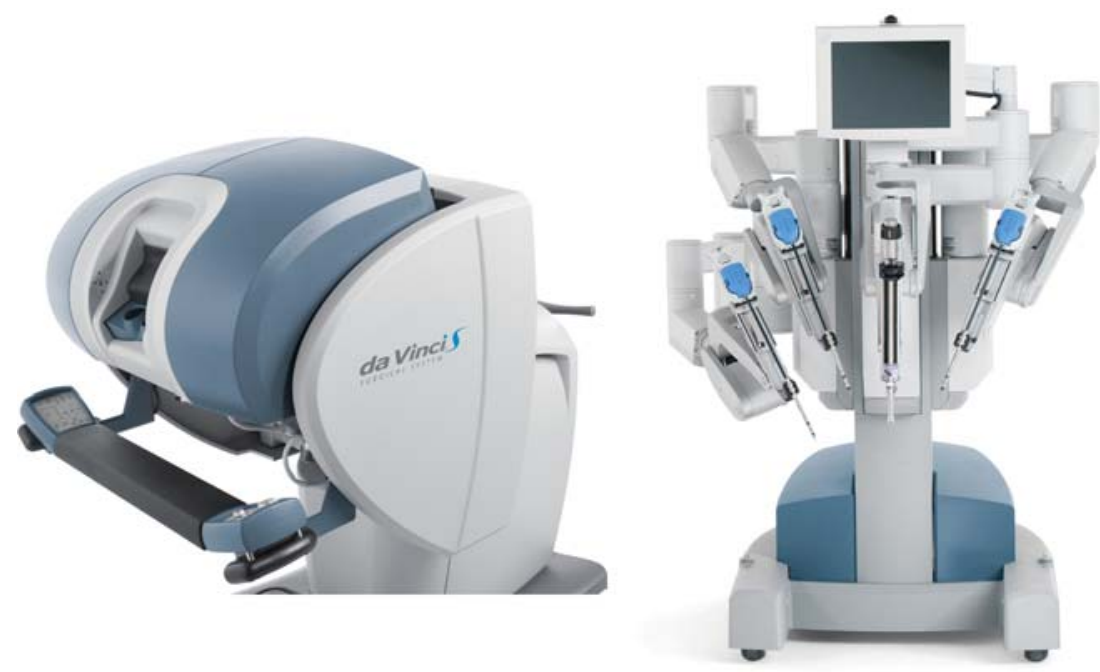

The daVinci Surgical System with the surgeon's console to the left and the robotic arm tower on the right. bombs, surveying space and the deep sea, and treating patients on the battlefield from a safe haven behind the front include just a few potential applications. Telepresence, or the insertion of the robot operator, into a virtualreality display emerged from these visions of potential benefit. In the 1980s the National Aeronautics and Space Administration (NASA) joined with the Ames Research Center (Palo Alto, CA, USA) to begin the development of a headmounted virtual-reality display to allow users to immerse themselves in the large data sets that were transmitted from aerospace missions. By coupling 3D stereoscopic vision with the DataGlove (VPL Research, Inc, Redwood City, CA, USA), users could see their own interactions with a virtual world $[5,13]$.

The potential advantages that telepresence could provide surgeons were recognized by Scott Fisher, Ph.D. (a NASA scientist) and Joe Rosen, MD (a Stanford University, Palo Alto, CA plastic surgeon). They envisioned telepresence surgery to involve the virtual insertion of the surgeon into the operative field with the manipulation of remote robotic arms. Fisher and Rosen collaborated with Phil Green, Ph.D. of the Stanford Research Institute (now SRI International, Menlo Park, CA, USA) to develop a robotic arm. Over the next decade, the field of telerobotic surgery grew and the concept of integrating this technology into the burgeoning field of laparascopic surgery was fully realized [13]. The concept was introduced to the Pentagon's Defense Advanced Research Projects Agency (DARPA) with the goal of allowing a surgeon to treat a wounded soldier on the battlefield from a remote safe haven-with the surgeon's hands controlling robotic arms on the battlefield $[5,14,15]$.

Initially funded by DARPA, Computer Motion, Inc. (acquired in 2003 by Intuitive Surgical, Sunnyvale, CA, USA) developed the automated endoscopic system for optimal positioning (AESOP) system, a robotic arm for endoscopic camera control. AESOP, designed to replace a surgical assistant in laparoscopic surgery, was coupled with the Hermes voice-activation system to allow endoscope control by voice command [15]. These devices achieved FDA approval in 1994. While the AESOP/Hermes platform was the first actively marketed telerobotic manipulators system, the devices' most significant function was serving as the groundwork for the surgical robotic devices currently integrated into clinical practice.

In 1995 the licensing rights for the original SRI telepresence surgical system were acquired by Fredrick Moll MD, Robert Younge and John Freund MD, and Intuitive Surgical, Inc. was formed. The potential for widespread clinical application of the newly developed telerobotic devices was commercially recognized and in 1997, Intuitive Surgical's daVinci surgical system was used to perform a laparoscopic cholecystectomy in Belgium [15]. The original daVinci surgical system consisted of a remote surgeon's console and a three-armed robotically controlled instrument drive system (Fig. 2). In the surgeon's console are two viewers, one for each eye, which provide a threedimensional view of the operating field. The surgeon sits at the console resting his hands in control grips which allow for arm, wrist, and pincer movement (Fig. 3). The surgeon's hands, which rest in line with the visual axis, control the seven degrees of freedom of the wristed instrumentation (Fig. 4). The robotically controlled instrument drive system is a tower with three multiply jointed arms, two of which control a variety of $8 \mathrm{~mm}$ surgical instruments while the third drives a binocular video endoscope (Fig. 5). The video system provides $10 \times$ to $15 \times$ magnification and true $3 \mathrm{D}$ vision. The wristed instruments track the surgeon's movements 1,300 times per second and 


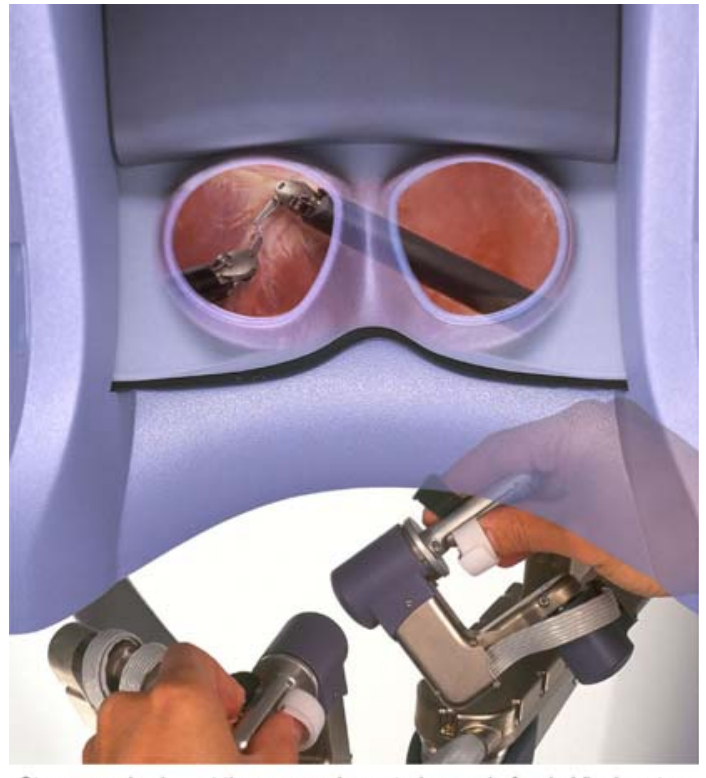

Stereoscopic view at the surgeon's control console for da Vinci system.

Fig. 3 The surgeon's hands rest in grips that are in line with the visual axis of the binocular viewers

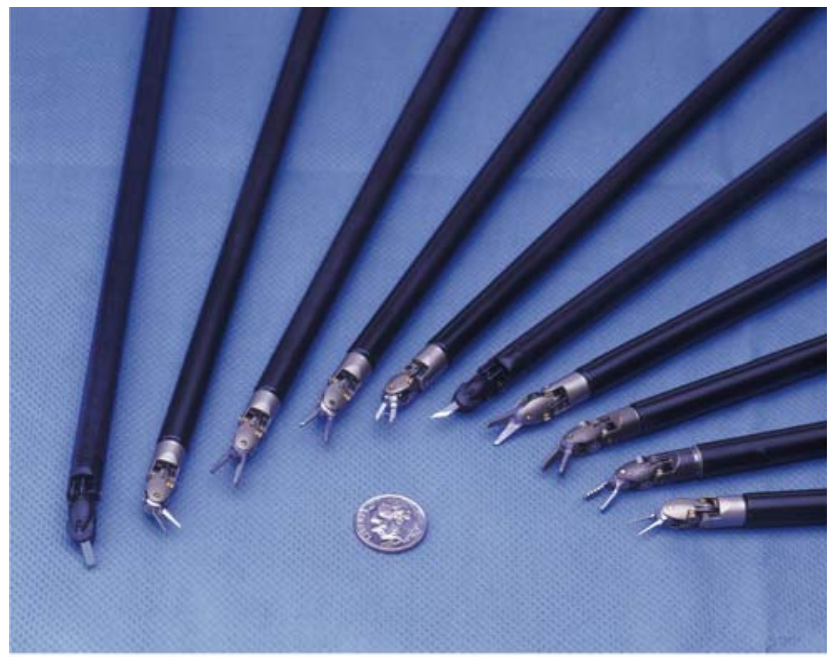

$8 \mathrm{~mm}$ "Endowrist" instruments compatible with the daVinci Surgical System.

Fig. 4 A variety of the $8 \mathrm{~mm}$ endowristed instruments

provide for tremor filtration and scaled motion-translating larger movements of the surgeon's hand into finer movements of the wristed instrumentation.

In 1999, Computer Motion Inc. introduced the Zeus surgical system, which differed from daVinci primarily in the configuration of the surgeon's workstation (Fig. 6). To operate the Zeus, the surgeon sits at a console and wears polarized goggles to view the operative field in 3D. Computer Motion Inc. was acquired by Intuitive Surgical in 2003 and the Zeus system is no longer commercially available.

The Zeus surgical system was used in 2001 for the first telepresence surgical procedure. The military's

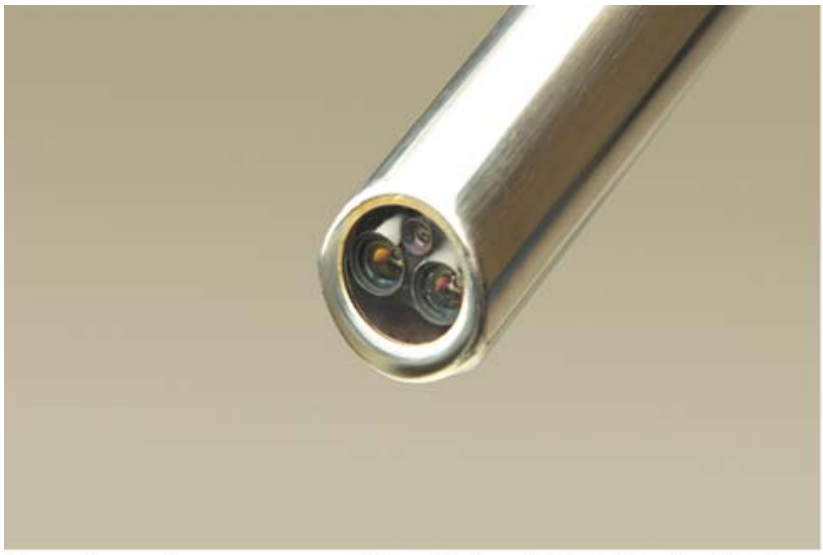

Binocular endoscope compatible with the daVinci Surgical System

Fig. 5 The binocular endoscope places two glass rods in line, with each sending an image to a distinct camera head

vision for telepresence surgery was realized when the first transatlantic surgical procedure, a laparoscopic cholecystectomy, was performed on a patient in Strasbourg, France by a surgeon seated at a console 3,800 miles away in New York, United States. Utilizing a $155 \mathrm{~ms}$ bandwidth, the time delay between the operating surgeon's movements and the remote instrument movement was minimized.

Since the original introduction of the daVinci surgical system, there have been several modifications. A fourth robotic arm has been added which allows the surgeon to toggle between three instruments. An increasing number of both 8 and $5 \mathrm{~mm}$ surgical instruments are available and the new daVinci S (Intuitive Surgical) adds an interactive video displays and more streamlined setup.

The daVinci surgical system has now been FDA approved for a variety of general, cardiac, gynecologic, and urologic procedures. Clinical data measures document equal or improved surgical outcomes with improved postoperative function, decreased blood loss, shorter hospital stays, and a favorable learning curve for newly trained robotic surgeons [16-19]. Over 500 daVinci surgical systems have been installed worldwide and device use continues to increase. Procedure development in the thoracic and abdomino-pelvic surgery continues as does clinical research into applications in the upper aerodigestive tract, skull base, and soft tissues of the neck [20-23].

\section{Future developments}

The currently available surgical robots, in both clinical use and clinical trials offer potential advantages to truly recognize the concept of minimally invasive surgery. Robotic devices with more-streamlined platforms, smaller instrumentation, and remote telementoring will all likely be a reality in the foreseeable future. 
Fig. 6 The Zeus surgical system with its table-mounted arms (left) and the surgeon's console (right)

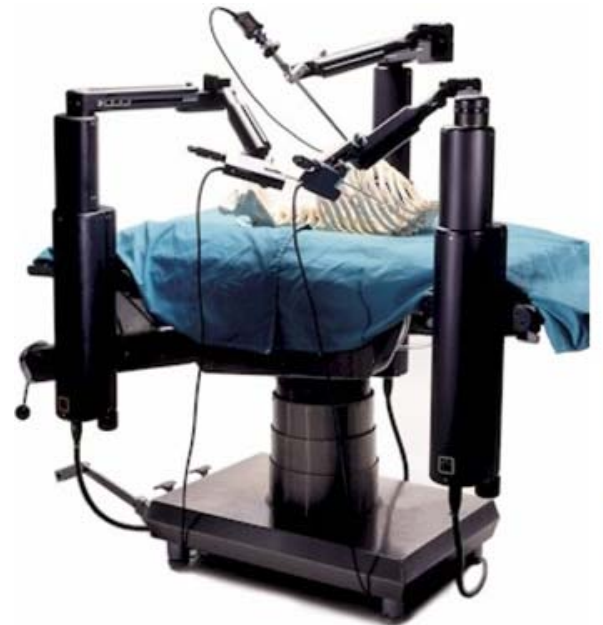

The Zeus Surgical System

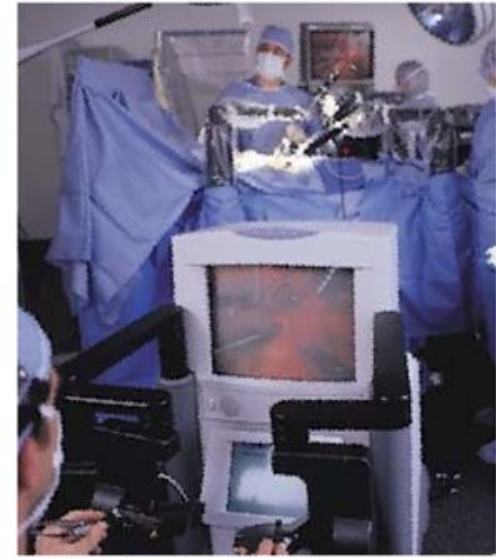

View from Surgeon's Console, Zeus
Current and previously marketed telerobotic devices lack haptic or sensory feedback. While this technology has been the source of a great deal of research and funding, with numerous patents having been granted, it has not been applied clinically [24]. Arguments against the need for haptic feedback include: (1) the substitution of improved optics that offer visual cues to the force-response relationships between the surgeon and the tissues, and (2) the lack of haptics in conventional endoscopic surgery, which uses long rigid instrumentation. However, the potential to improve upon current technology by the addition of haptic feedback exists and will likely continue to be a source of additional research.

\section{Conclusions}

While the concept of robotics began as science fiction, today robots play important roles in modern life. In the healthcare industry, surgical robotic promise to play a more integral role in the years to come. From the original concept of battlefield surgery by DARPA to the transatlantic laparoscopic cholecystectomy, great advances have been made. Following the tenets of modern and ancient medicine including clinical outcomes research and "do[ing] no harm", robotic surgery, both with active surgical robotic mills and telerobotic manipulators, has the potential to offer patients the opportunity to optimize minimally invasive surgery.

\section{References}

1. Capek J (1925) Opilec. In: Lelio A Pro Delfina. Aventinum, Prague

2. Capek K (2004) R.U.R. (Rossum's Universal Robots). Penguin Group, New York
3. Capek K (1923) The Meaning of R.U.R. Saturday Review July 21, 136:79

4. Asimov I (1942) Runaround. In: Astounding Science Fiction March

5. Gourin CG, Terris DJ (2006) History of robotic surgery. In: Faust RA (ed) Robotics in surgery: history, current and future applications. Nova Science, New York (in press). ISBN 1-60021-386-1

6. http://www.robothalloffame.org/unimate.html. Accessed on 10/ $13 / 2005$

7. http://www.biomed.brown.edu/Courses/BI108/BI108_2005_Gro ups/04/neurology.html. Accessed on 10/13/2005

8. Nishihara S, Sugano N, Nishii T, Tanaka H, Nakamura N, Yoshikawa H, Ochi T (2004) Clinical accuracy evaluation of femoral canal preparation using the ROBODOC system. J Orthop Sci 9(5):452-461

9. Honl M, Dierk O, Gauck C, Carrero V, Lampe F, Dries S, Quante M, Schwieger K, Hille E, Morlock MM (2003) Comparison of robotic-assisted and manual implantation of a primary total hip replacement. A prospective study. J Bone Joint Surg 85$\mathrm{A}(8): 1470-1478$

10. Zipper SG, Puschmann H (2005) Nerve injuries after computer-assisted hip replacement: case series with 29 patients. German. Zeitschrift Orthopadie Ihre Grenzgebiete 143(4):399-402

11. Cobb J, Henckel J, Gomes P, Harris S, Jakopec M, Rodriguez F, Barett A, Davies B (2006) Hands-on robotic unicompartmental knee replacement: a prospective, randomized controlled study of the acrobat system. J Bone Joint Surg 88(2):188-197

12. Federspil PA, Geisthoff UW, Henrich D, Plinkert PK (2003) Development of the first force-controlled robot for otoneurosurgery. Laryngoscope 113(3):465-471

13. Satava RM (2002) Surgical robotics: the early chronicles. Surg Laparosc Endosc Percutan Tech 12(1):6-16

14. Gourin CG, Terris DJ (2004) Surgical robotics in otolaryngology: expanding the technology envelope. Curr Opin Otol Head Neck Surg 12(3):204-208

15. Ballantyne GH (2002) Robotic surgery, telerobotic surgery, telepresence, and telementoring. Surg Endosc 12(1):6-16

16. Patel VR, Tully AS, Holmes R, Lindsay J (2005) Robotic radical prostatectomy in the community setting - the learning curve and beyond: initial 200 cases. J Urol 174(1):269-272

17. Nifong LW, Chitwood WR, Pappas PS, Smith CR, Argenziano M, Starnes VA, Shah PM (2005) Robotic mitral valve surgery: a United States multicenter trial. J Thorac Cardiovasc Surg 129(6):1395-1404

18. Menon M, Tewari A, Peabody JO, Shrivastava A, Kaul S, Bhandari A, Hemal AK (2004) Vattikuti Institute prostatectomy, a tech- 
nique of robotic radical prostatectomy for management of localized carcinoma of the prostate: experience of over 1100 cases. Urol Clin North Am 31(4):701-717

19. Kypson AP, Nifong LW, Chitwood WR Jr (2003) Robotic mitral valve surgery. Surg Clin North Am 83(6):1387-1403

20. Hockstein NG, Nolan JP, O'Malley BW, Woo YJ (2005) Robotassisted laryngeal and pharyngeal microsurgery: results of robotic cadaver dissections. Laryngoscope 115(6):1003-1008

21. Lobe TE, Wright SK, Irish MS (2005) Novel uses of surgical robotics in head and neck surgery. J Laparoendosc Adv Surg Tech A 15(6):647-652
22. O'Malley BW, Weinstein GS, Snyder W, Hockstein NG (2006) Transoral robotic surgery (TORS) for base of tongue neoplasms. Laryngoscope 116(8):1465-1472

23. Terris DJ, Haus BM, Gourin CG, Lilagan PE (2005) Endo-robotic resection of the subandibular gland in a cadaver model. Head Neck 27(11):946-951

24. Madhani AJ, Niemeyer G, Salisbury JK (1998) The black falcon: a teleoperated surgical instrument for minimally invasive surgery. In: Proceedings IEEE/Robotics Society of Japan International Conference on Intelligent Robotic Systems, vol 2, pp 936-944 